\title{
Resistance of a free operant to extinction and suppression with punishment as a function of amount of training,'
}

\author{
DAVID G. BORH \\ UNIVERSITY OF UTAH
}

Three groups of 20 Ss were given 200, 1,000 or 3,000 reinforcements in a Skinner box on a VI 30-sec. schedule of reinforcement. On the day following completion of training, half of each group was exposed to a $1 \mathrm{hr}$. test session in which punishment followed each response; the remaining $S$ s were given a $1 \mathrm{hr}$. extinction test session. The mean number of punished responses during testing was nonmonotonically related to amount of training, while additional training increased resistance to extinction of the unpunished groups.

Recent experiments on the effects of amount of training have shown that under some conditions responses highly overtrained may be more easily eliminated than responses less completely trained. This phenomenon has been observed following nondiscriminative training in the runway under conditions of punishment (Miller, 1960; Karsh, 1962) and extinction (e.g., North \& Stimmel, 1960; Siegel \& Wagner, 1963). Following nondiscriminative training in the Skinner box, on the other hand, the overtraining effect appears to have not been demonstrated under conditions of extinction (see Sperling, 1965) or punishment (Lawson \& Born, 1964). However, in the Lawson and Born study, the punishment was sufficiently intense that few responses occurred before Ss in all groups met the suppression criterion, and amount of training appeared to be unrelated to ease of suppression with punishment; of particular interest is that time to recovery from punishment was nonmonotonically (generally U-shaped) related to amount of training. Using a milder punishing stimulus, the present experiment attempts to reevaluate the relationship between amount of nondiscriminative training in the Skinner box and ease of suppression with punishment, and provides further data relating the effects of amount of training to resistance to extinction.

Method

The Ss were 60 male Sprague-Dawley albino rats between 150-200 days of age reduced to $80 \%$ of free feeding body weight. The apparatus consisted of two Plexiglas chambers 12 in. long, 8 in. wide, and 10-1/2 in. high, equipped with a Grason-Stadler lever, Davis Feeder (Model 109), and a grid floor constructed of $1 / 8$ in. brass rods centered $1 / 2$ in. apart. Punishment was provided by a $150 \mathrm{~V}$ ac shock which passed through a $110 \mathrm{~K}$ ohm resistor in series with $\mathrm{S}$. The chambers were located in a different room than the control and recording equipment, and each chamber was housed in a nonfunctional refrigerator for sound attenuation.

The experiment was run in two replications, 30 Ss per replication. In the first replication, training started at the same time for all groups and testing occurred at different times. In the second replication, the restricted feeding regimen was imposed upon all Ss the same day, but the start of training was postponed for the 200 and 1000 reinforcement groups so that all Ss could be tested at approximately the same time.

Each S was randomly assigned to punishment or extinction groups, and to one of three levels of training $(200,1000$, or 3000 reinforcements) under the restriction that an equal number of Ss be run under each condition within each replication. Potential differences between the two chambers were counterbalanced across replications.

Pretraining consisted of five sessions of magazine training for each $S$ with the lever inoperative; $5045 \mathrm{mg}$ Noyes pellets were delivered on a variable interval (VI) 1 min. basis in each session. During the sixth session each $\mathrm{S}$ was placed in his experimental chamber and each of the first 25 lever presses produced a food pellet; for the remainder of the session ( 75 additional reinforcements) a VI 30 sec. reinforcement schedule was in effect. Six Ss did not begin lever pressing within approximately $20 \mathrm{~min}$. and were shaped to press. None of these Ss required more than 12 shaping reinforcements. VI 30 sec. training continued daily, 100 reinforcements per session, until S received either 200,1000 , or 3000 reinforcements for lever pressing, depending upon the training level to which he had been assigned.

On the day following completion of training, each $S$ in the punishment group was placed in his experimental chamber and each lever press produced a shock of $1 \mathrm{sec}$. duration through the grid floor. No food pellets were delivered during this session. The punishment contingency remained in effect for $1 \mathrm{hr}$., or until s failed to respond for $30 \mathrm{~min}$. if suppression was not complete at the end of $1 \mathrm{hr}$. Number of responses to both suppression criteria were recorded for each $\mathrm{S}$.

Ss in the corresponding unpunished group remained in their home cages on the day experimental Ss were being punished since the punishment sessions were very long for some Ss. ${ }^{3}$ On the second day after completion of training, each unpunished $S$ was placed in the experimental chamber where he remained for $1 \mathrm{hr}$. Lever presses during this period produced neither food nor shock, and total number of responses was recorded for eacn $\mathrm{S}$.

Resulis

One $\mathrm{S}$ in the punishment group receiving 3000 rein- 
forcements (first replication) was discarded because of an experimental error. For purposes of statistical analysis that $S$ was assigned the mean score of the other Ss comparably treated. Also, one S in the 200 reinforcement punishment group (second replication) was removed from the chamber upon meeting the $30 \mathrm{~min}$. suppression criterion, but approxdmately $10 \mathrm{~min}$. before completion of the $1 \mathrm{hr}$. session. The score based on a $50 \mathrm{~min}$. session was retained for this $S$ since a preliminary experiment had shown that the criterion represented virtually complete suppression on the test day.

Figure 1 shows the mean number of responses made by both punished and unpunished groups during the $1 \mathrm{hr}$. test session as a function of amount of training. Since within cell variances for punished and unpunished groups were markedly different, the data for the two conditions were subjected to independent 3 by 2 (training levels by replications) analyses of variance. The analysis of number of punished responses revealed a significant effect of training levels $(F=4.20, d f=2 / 23, p<.05)$. Subsequent $t$ tests showed the 200 and 3000 reinforcement group means were statistically different from the 1000 group mean ( $p<.01$ in each case), although they did not differ reliably from each other $(p>.05)$. A similar analysis of responses to the $30 \mathrm{~min}$. suppression criterion (the criterion used by Lawson \& Born) resulted in identical statistically significant differences. The analysis of number of responses made by the unpunished groups also revealed a significant effect of training levels ( $F=6.25$, df $=2 / 24, p<.01$ ). However, in this case $t$ tests showed the 200 reinforcement group was significantly different from the other two groups ( $p<.01$ in each case), although the latter groups did not differ reliably $(p>.05)$.

\section{Discussion}

The obtained nonmonotonic relationship between amount of training and ease of suppression with punishment extends the overtraining punishment effect to the free operant situation following nondiscriminative training. As mentioned earlier, a similar finding was obtained in the runway situation by Miller (1960) and Karsh (1962). Thus, the overtraining punishment effect appears to transcend inherent differences between the runway and the Skinner box, and may therefore occur across a wider range of training and testing conditions than the overtraining extinction effect. Additionally, it may be noted that in the studies of Miller and Karsh, punishment was administered on rewarded trials; in the present experiment punishment was administered during extinction.

Perhaps the most interesting general outcome of the present experiment is apparent from a comparison of the punished and unpunished groups in Fig. 1. To the extent that ease of suppression with punishment and simple resistance to extinction are equivalent indices of response strength, both should yield the same functional relationships. The data in Fig. 1 suggest they are not equivalent indices of the effects of overtraining. The present results also indicate that overtraining effects

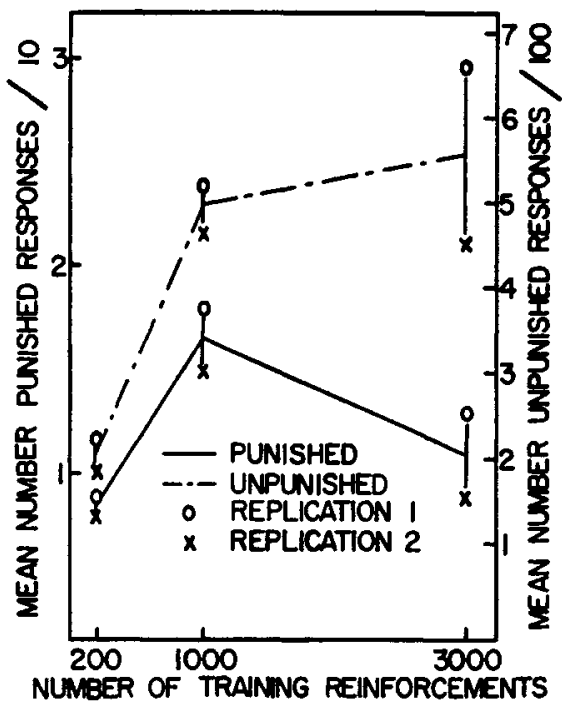

Fig. 1. Mean number of responses during testing for punished and unpunished groups as a function of replication procedure and amount of training.

must be viewed as an interaction of training variables and the conditions of testing; i.e., test conditions are not simply a passive medium through which the effects of training variables become manifest. From these data it appears that variables in addition to amount of reward (Theios \& Blosser, 1965) may interact with extended training to facilitate response elimination during testing.

\section{References}

Karsh, Eileen. Effects of number of rewarded trials and intensity of punishment on running speed. J. comp. physiol. Psychol., $1962,55,44-51$.

Lawson, R., \& Bom, D. G. Recovery from punishment as a function of number of pre-punishment reinforcements. Psychon. Sci., 1964, 1, 269-270.

Miller, N. E. Learning resistance to pain and fear: Effects of overlearning, exposure, and rewarded exposure in context. $J$. exp. Psychol., 1960, 60, 137-145.

North, A. J., \& Stimmel, D. T. Extinction of an instrumental response following a large number of reinforcements. Psychol. Rep., 1960, 6, 227-234.

Siegel, S., \& Wagner, A. R. Extended acquisition training and resistance to extinction. J. exp. Psychol., 1963, 66, 308-310. Spering, Sally E. Reversal learning and resistance to extinction: A review of the rat literature. Psychol. Bull., 1965, 63, 281-297.

Theios, J., \& Blosser, D. The overlearning reversal effect and magnitude of reward. J. comp. physiol. Psychol., 1965, 59, 252-257.

\section{Notes}

1. This research was supported by a grant from the University of Utah Faculty Research Fund.

2. The writer expresses his gratitude to John Behney, Ronald Menlove, and James Peterson for assistance in running Ss.

3. While the difference in day of testing does not make test conditions strictly comparable for the punished and unpunished groups, it seems highly unlikely that the general form of either of the obtained functions would be markedly changed in the absence of this difference. 